\section{Paediatric crossword puzzle 26}

Manouri P Senanayake ${ }^{1}$

Sri Lanka Journal Of Child Health, 2015; 44(1): 67

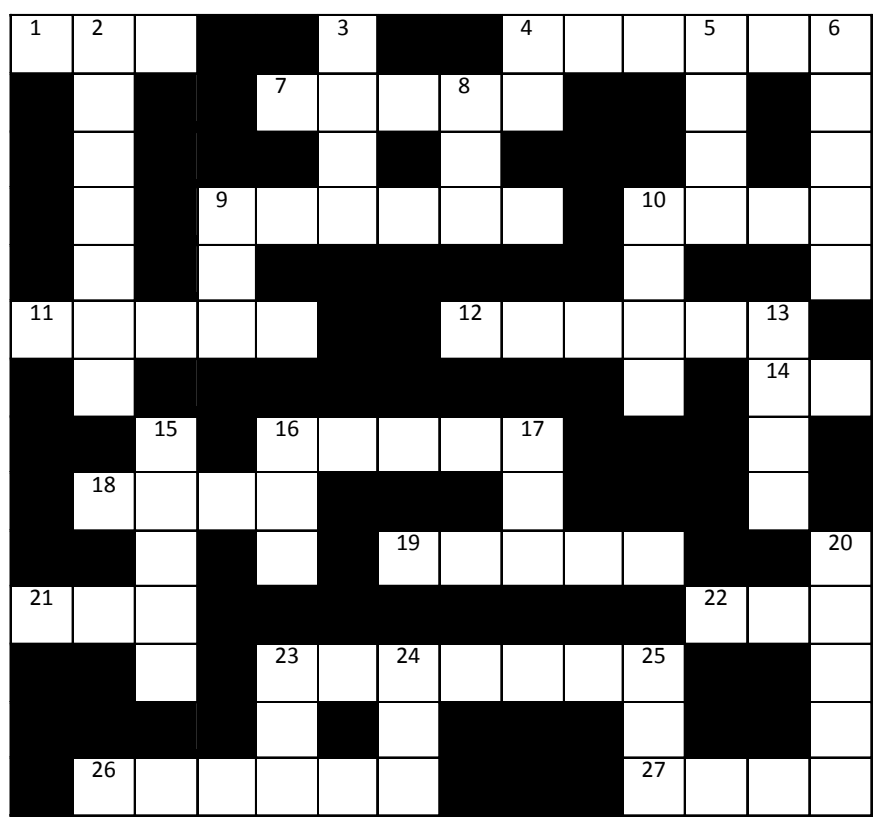

ACROSS

1. Current outbreak of Ebola was started by a ------- bite

4. Second carpal bone to appear

7. Outer ear

9. Impairs social interaction

10. Not eliminated with Pyrantel(---- worm)

11. Capitate is the-----carpal bone to appear

12. A common cause of acute ataxia in children

14. West syndrome (syn)

16. Ebola is named after a in Zaire

18. Drug metabolism is also called biotic metabolism

19. Experimental drug against Ebola

21. Is not invariably present in antiphospholipid syndrome

22. Commonest childhood malignancy

23. Word "Denga" is from this language

26. Current outbreak of Ebola started in

27. Number of main blood groups

\section{DOWN}

2. Treats antiphospholipd syndrome

3. Herb with proven medicinal value for irritable bowel syndrome

5. Does not control aldosterone secretion

6. Dental research claims delay due to neonatal illness

8. Pervasive developmental disorder (PPD) featuring between Aspergers and Autism

9. Causes recurrent thrombosis and fetal loss(abbv)

10. A flying insect that injects poison

13. A clotting factor present in cryo precipitate

15. "There will be no dengue if there is no

16. Bevacizumab therapy is better than laser therapy for

17. Assay technique that measure hormones using antibodies

20. Umbilical granuloma drains fluid

23. Childhood exposure determines skin cancer

24. "-----." is defined as maternal age $>35$ years(abbr)

25. Cause of increased rate of twinning (abbv) 